\title{
Piloting a national laboratory electronic programme status reporting system in Ekurhuleni health district, South Africa
}

\author{
N Cassim, MPH; L M Coetzee, PhD; D K Glencross, MB BCh, MMed (Haematology) \\ National Health Laboratory Service, National Priority Programme, Johannesburg, South Africa, and Department of Haematology \\ and Molecular Medicine, Faculty of Health Sciences, University of the Witwatersrand, Johannesburg
}

Corresponding author: N Cassim (naseem.cassim@nhls.ac.za)

\begin{abstract}
Background. The National Health Laboratory Service (NHLS) performs $\sim 4$ million CD4 tests per annum for the public health sector at $61 \mathrm{CD} 4$ testing laboratories across South Africa. Currently, CD4 laboratory data captured do not differentiate between antiretroviral treatment (ART) and pre-ART care.

Methods. A cross-sectional study was undertaken to evaluate a redesigned Comprehensive Care, Management and Treatment of HIV and AIDS (CCMT) request form, incorporating a two-tick collection procedure linking the CD4 test request to patient CCMT programme status. Field testing was undertaken at three health facilities, where healthcare personnel were required to capture whether the CD4 count requested was a 'first-ever CD4', 'CD4 taken previously, not yet in ART care' or 'in ART care'. All data were extracted from the NHLS Corporate Data Warehouse and analysed using Microsoft Excel and Stata-12.

Results. A substantial increase in the number of request forms with a CCMT programme status $(28.1 \% \mathrm{v} .84 .4 \%)$ was reported pre- and post-implementation. Post-implementation data $(N=1004)$ revealed that $30.8 \%$ patients were ART naive ('first-ever CD4'), with $7.4 \%$ 'not yet on ART' (median CD4 counts of 150 and 328 cells $/ \mu \mathrm{L}$, respectively). Patients on ART comprised $61.9 \%$ of the study group (median CD4 count $\sim 346$ cells $/ \mu \mathrm{L}$ ). Sixty percent of patients were aged between 30 and 44 years, and females predominated (male/ female ratio 0.7:1).

Conclusions. A simple modification to the CCMT request form can successfully facilitate collection of programme status. For national implementation, it would be advantageous to have a unique patient identifier to further enhance laboratory-based programmatic monitoring and evaluation.
\end{abstract}

S Afr Med J 2016;106(4):374-377. DOI:10.7196/SAMJ.2016.v106i4.10066

HIV infection and AIDS comprise one of the main public health challenges facing South Africa (SA). In response to the epidemic, the National Department of Health (NDoH) initiated the Comprehensive Care, Management and Treatment of HIV and AIDS (CCMT) programme in 2004. In 2007, the NDoH outlined its plans to extend the HIV \& AIDS and STI Strategic Plan for South Africa 2007 - 2011 (NSP). ${ }^{[1]}$ The primary aim of the NSP was to reduce the prevalence of HIV by $50 \%$ and to expand access to treatment, care and support to $80 \%$ of people who need it by 2011 and beyond. ${ }^{[2]}$ In August 2011, the NDoH released the new NSP for the period $2012-2016 .{ }^{[3]}$ The key goals set by the NSP for 2012 - 2016 were to reduce the rate of new HIV infections and HIVassociated maternal mortality by $50 \%$ as well as reducing new HIV infections in children by $90 \% .{ }^{[3]}$

In support of this programme, the National Health Laboratory Service (NHLS) provided 3.9 million CD4 tests during 2013/14, through a network of 61 laboratories across SA. ${ }^{[4]}$ Analysis of CD4 laboratory data provides important information on the CCMT programme, reported monthly to the NDoH, including the median CD4 counts for various age groups, with the related test request volumes across districts. Currently, however, information from sample request forms does not include information to identify the proportion of patients accessing care for the first time v. those who are already enrolled into care and being followed up, or alternatively are in wellness programmes waiting to qualify for treatment. This information is critical to determine the impact of HIV programmes on patient initiation, and to assist in management of patient monitoring and access to care.

\section{Objectives}

The objectives of this study were threefold. Firstly, the usefulness of introducing a 'two-tick' system on an improved test request form, linked to CD4 testing, was explored for linking the CD4 count to patient CCMT programme status. This made it possible to identify treatment-naive patients (first-time CD4 counts) from those in staging and already enrolled for antiretroviral treatment (ART). Secondly, the proportions of patients in the pre-ART and ART programme status categories (as well as within specific age groups) as a percentage of total CD4 samples received could be analysed to assess overall numbers of specific patient groups who are accessing and/or eligible for treatment (or not) in a given healthcare facility. Thirdly, the median CD4 counts within groups characterised by this two-tick system could be used to provide information about overall patient wellness according to CCMT status provided within defined age groups.

\section{Methods}

A cross-sectional study was designed to evaluate CCMT data collection enabled by introduction of a new data collection instrument (a redesigned CCMT request form) that was introduced at three HIV clinics in Ekurhuleni Metropolitan Municipality, Gauteng Province, SA (the study sites were Tambo Memorial, Far East Rand and Tembisa). A MaCorr sample size of $1200 \mathrm{CD} 4$ samples was calculated for the study, with 500 collections planned for the Tambo Memorial study site and 350 each for the Far East Rand and Tembisa. Samples from patients $<18$ years of age were excluded.

To assess the success of the implementation, CD4 data were extracted from the NHLS Corporate Data Warehouse (CDW) for 2 months (June 
and July 2012) prior to implementation of the new form, and data were analysed using Excel and Intercooled Stata 11. This analysis enabled an assessment of baseline CCMT data captured before introduction of the new form. The new request form was designed to incorporate two additional sets of tick blocks that were added onto the existing NHLS request form, in close proximity to the $\mathrm{CD} 4$ test request tick box. By ticking a tick block linked to the CD4 test request, a healthcare worker was able to provide specific information about the patient's related CCMT programme status. Available options were 'first-ever CD4' count, 'CD4 taken previously, not yet in ART care' and 'in ART care. New data fields were established on the NHLS Laboratory Information System (LIS) to facilitate the data capture of the three new CCMT status parameters. CD4/CCMT data were categorised to identify forms on which the required information was incorrectly filled out or not filled out at all. Data capture rates before (number of sample data entries with any relevant linked CCMT information before implementation) and after implementation of the new data collection form were calculated. Only LIS/CDW CD4 data entries that were linked to the new CCMT test request form (identified with a custom barcode sequence) were included in the 'post-implementation' analysis data set.

After collection of LIS data through CDW, the post-implementation $\mathrm{CD} 4$ and CCMT data were organised according to CCMT programme status. Median CD4 counts were calculated for each group and the proportion of samples established according to gender, specified antenatal survey age groups ${ }^{[5]}$ and predefined $\mathrm{CD} 4$ range distributions. The latter was based on previous and current treatment thresholds to indicate the level of wellness/ immune deficiency per subgroup analysed. ${ }^{[6]}$

\section{Results}

During the study period, redesigned CCMT request forms were received with $1266 \mathrm{CD} 4$ samples from the three participating study sites, reaching $99 \%$ of the target sample size. Seventy-six CD4 sample results $(6.0 \%)$ from children aged $<18$ years were excluded, leaving $1190 \mathrm{CD} 4$ data entries for final analysis $(94.0 \%)$. The contribution analyses of the three clinics showed that $46.8 \% \quad(n=557)$ of CD4 sample requests were received from the Tambo Memorial study site, $31.8 \%$ $(n=378)$ from Tembisa and the remaining $21.4 \%(n=255)$ from the Far East Rand.

CCMT programme status data were not captured on 186/1 190 (15.6\%) of new request forms received. In 165/186 (88.7\%), no information/programme status was captured on the LIS. In $17 / 186$ instances (9.1\%), the CCMT information was incorrectly captured by the LIS data clerk and an invalid option was provided in the programme status field. These were categorised as 'programme status not relevant to study', and comprised entries such as '3TC' $(n=4)$, 'D4T' $(n=1)$, 'adverse effect' $(n=4)$, 'non-adherence' $(n=1)$, 'virological failure' $(n=1)$ and 'toxicity' $(n=6)$.

In $4 / 186$ instances (2.2\%), the healthcare worker did not check any of the new request form tick blocks; these were identified and entered onto the NHLS LIS by the NHLS data clerks as 'no details supplied?

Table 1 outlines the impact of the new form and related new three-option tickblock system, compared with the preimplementation proportion of existing request forms with CCMT data captured. Despite the fact that $15.6 \%$ of new forms were incorrectly or not filled out, the linked tick-block system on the new CCMT form enabled a $>300 \%$ increase in the number of forms received with relevant useful patient CCMT data.

Fig. 1 outlines analyses performed on the remaining data $(n=1004)$, characterised

Table 1. CCMT programme status data capture rates before and after the study

\begin{tabular}{lllll}
\hline & $\begin{array}{l}\text { Samples with } \\
\text { CCMT programme } \\
\text { status, } \boldsymbol{n}\end{array}$ & $\begin{array}{l}\text { Samples without } \\
\text { CCMT programme } \\
\text { status, } \boldsymbol{n}\end{array}$ & $\begin{array}{l}\text { \% of samples with } \\
\text { CCMT programme } \\
\text { status }\end{array}$ \\
\hline Pre-study & 873 & 2230 & 3103 & 28.1 \\
Study & 1004 & 186 & 1190 & 84.4 \\
\hline
\end{tabular}

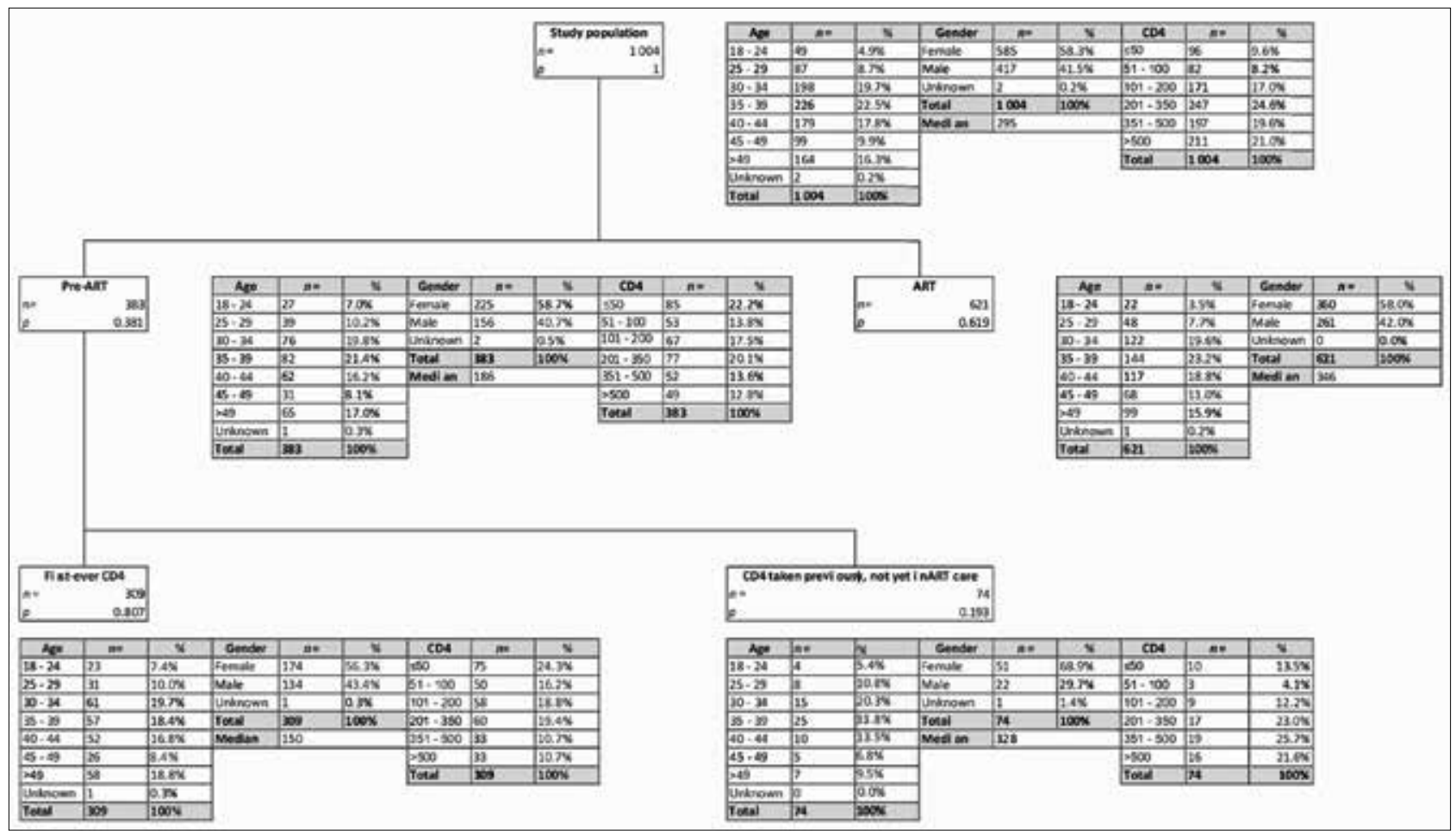

Fig. 1. CD4 test range, age and gender distribution for each CD4 CCMT programme status. 
according to CCMT status. The latter included: (i) 'pre-ART', which was further defined as 'first-ever CD4' or 'CD4 taken previously, not yet in care'; or (ii) 'ART'. Data were further categorised by specific age categories as described above, as well as by $\mathrm{CD} 4$ count threshold, including $<50,100,200,350$ and $<500$ cells $/ \mu \mathrm{L}$ categories.

Patient gender information was captured for $99.8 \%$ of CD4 samples, $58.3 \%$ from female patients $(n=585)$ and $41.6 \%(n=417)$ from males (ratio $\sim 3: 2$ ). A similar gender proportion was noted across all CCMT categories (Fig. 1), with the exception of the group 'CD4 taken previously, but not yet in care', where the ratio of female to male patients more than doubled, increasing to $\sim 7: 3$. The majority of CD4 samples received were from patients aged between 30 and 44 years $(60.1 \%)$, with $13.5 \%$ and $26.4 \%$ of samples from patients aged 18 - 29 years and $>44$ years, respectively. These proportions were similar across all CCMT groups.

Of the CD4 samples, $38.1 \%(n=383)$ were identified as 'preART', 'first-ever CD4' CCMT programme status comprised $30.8 \%$ $(n=309)$, and 'CD4 taken previously, not yet in ART care' comprised $7.4 \%(n=74)$. A summary of age and CD4 range distribution is shown in Fig. 1. In the treatment-naive patient group ('first-ever CD4'), $89.3 \%$ of patients were eligible for treatment at the current 2015 treatment guideline threshold of $500 \mathrm{CD} 4$ cells $/ \mu \mathrm{L}^{[6]}$ The majority of the patients in this group had a median CD4 count of 150 cells $/ \mu \mathrm{L}$. Overall, $78.6 \%$ overall had a CD 4 count $<350$ cells $/ \mu \mathrm{L}$; $59.2 \%$ of this group presented with a CD 4 count $<200$ cells $/ \mu \mathrm{L}$, while $40.5 \%$ were at risk for opportunistic cryptococcal infection, having CD4 counts $<100$ cells/ $\mu \mathrm{L}$. The group of patients awaiting enrolment into ART care ('CD4 taken previously, not yet in ART care'), had a slightly lower proportion of patients with very low CD4 counts (17.6\%), with a very high proportion eligible for ART at the current guideline threshold (78.4\%).

Patients identified as 'in ART care' comprised $61.9 \%$ of CD4 test requests overall $(n=621)$. Although a smaller proportion of patients could be categorised as markedly immunosuppressed (6.4\% had a CD4 count of $<100$ cells $/ \mu \mathrm{L}$ and $23.2 \%$ a count of $<200$ cells $/ \mu \mathrm{L}$ ), similar results for immunosuppressed patients awaiting enrolment into care were noted in this group. This was confirmed by the median CD4 count of 347 cells/ $\mu \mathrm{L}$, which was only slightly higher than the median of 324 cells $/ \mu \mathrm{L}$ noted in the 'awaiting enrolment' group.

\section{Discussion}

This study was designed to link a CD4 count to patient programme status by the simple addition of two tick boxes on an existing sample request form. Healthcare workers were prompted to provide additional relevant CCMT information, with minimal interruption to routine healthcare workflow. Compliance among healthcare workers providing this programme information increased from $28.1 \%$ to $84.4 \%$. It is clear that this simple modification to the request form was successful for three reasons. Firstly, the layout and placement of the relevant tick boxes meant that the healthcare worker was no longer required to search for the programme status fields located elsewhere on the (existing) request form, and the request for additional information was not ambiguous. Secondly, the direct linking and proximal placement of the CD4 test request to the tick block for programme status vastly improved data collection of relevant patient programme status. Thirdly, simplifying the data collection to two choices related to the timing of the CD4 test request and whether the patient was on treatment or not further encouraged compliance among attending healthcare personnel.

The successful use of this pilot request form, although limited to just 2 months of CCMT across three clinics in Ekurhuleni in Gauteng, had positive outcomes with important implications for collection and collation of national programme CCMT data. The simple directive of providing information about whether a CD4 count is 'first-ever' v. 'done previously' can be used to assess how many new patients are accessing HIV counselling and testing (HCT) and/or care and to ascertain the success of local programmes and associated proportions of patients who need fast-tracking and linkage to care (an aspect that is currently lacking in national reports). This information can be used to ensure that first-time HIV-positive patients start treatment as early as possible (according to threshold guidelines) to prevent late presentation, which is associated with opportunistic infections and hospitalisation.

In this study, a median CD4 count of 150 cells/ $\mu \mathrm{L}$ was noted in the group of patients having their first-ever CD4 count, indicating a markedly immunosuppressed group in need of urgent treatment intervention. Such low median presentation CD4 counts, similar to counts described locally ${ }^{[9]}$ and elsewhere in Africa, ${ }^{[7]}$ increase the likelihood of disease progression to AIDS, opportunistic infection and death if patients are not initiated on ART. ${ }^{[8]}$ According to the World Health Organization immunological classification for established HIV infection, ${ }^{[8]}$ this group has twice the risk of death or virological failure and may also be more likely to have opportunistic infections and other HIV-related conditions complicating presentation. Enabling these patients to be identified by linking programme status to CD4 results may allow for laboratory monitoring systems to be implemented to facilitate preselecting them for fast-tracking onto ART. There is an impression that the patients presenting for the first time are generally less ill than those seen at the start of the SA CCMT programme (monthly NHLS CDW CCMT reports, unpublished). However, lack of information and identification of patients with 'firstever CD4s' combined with at least a two-thirds majority enrolled onto treatment skews the overall median CD4 counts of this group, and gives the impression that CD4 counts of patients presenting for first-time HIV/CD4 testing have improved during the course of the CCMT programme. The data presented here show that people are still waiting until they feel ill before they present for HCT, with a median CD 4 count of $<200$ cells $/ \mu \mathrm{L}$ not vastly different from counts reported at the start of the CCMT programme. ${ }^{[9]}$

Furthermore, although it is commendable that a significant proportion of patients were already enrolled and 'in ART care' $(62.1 \%)$, it could not be ascertained from this study how long these patients had been enrolled in care. It is still worrying that overall lack of wellness and relative immunosuppression despite treatment still prevails in this group (median CD4 count just 347 cells/ $\mu \mathrm{L}$ ).

This study showed that mainly women presented for care. More than one-third had presented for HCT but were yet to start ART, and close to $90 \%$ of new patients were eligible for treatment. Of interest was the high proportion of females in the 'not yet enrolled in ART care' group. Reasons for the latter were not obvious.

The study confirmed that a simple modification to the existing request form can have a dramatic impact on how, and what, programmatic information is collected in relation to CD4 test requests. The challenge, however, is how this type of data collection tool can be scaled up to include other necessary related programme information (e.g. HIV viral load and screening for related HIV opportunistic infections/associated TB diagnostics) without making existing request forms too cumbersome with additional tick blocks. Unique patient identifiers are currently not used in our local healthcare databases despite use of unique identifiers, e.g. SA ID numbers, elsewhere in local government programmes to accurately collect and collate legitimate participant data (e.g. citizens who collect welfare grants are required to provide their SA ID numbers). ${ }^{[10]}$ Adopting the use of such master patient identifiers (MPIs) can have 
an immediate and dramatic impact on how local healthcare data are managed. Valuable information can be extracted immediately, without either necessitating modifications to request forms or developing indirect systems utilising less optimal methods that attempt to link and match variable patient identifiers to relevant diagnostic data, e.g. probabilistic matching in existing databases (personal communication, Dr Sergio Carmona, NHLS). Examples of where use of MPIs would significantly improve patient outcome include being able to track patients longitudinally by capture date and identify patients' 'first-ever' CD4 through to ART initiation (linked to 'first-ever' HIV viral load test requests), while additionally providing provincial and district alerts that could assist HIV programme co-ordinators in identifying patients who require immediate care (e.g. those with asymptomatic cryptococcal antigenaemia, where early intervention with fluconazole therapy can prevent progression to meningitis, potentially saving a life).

\section{Conclusion}

The challenges related to tracking patients from the point of entry at the time of HCT (denoted here as 'first-ever CD4') to enrolment onto a treatment programme (denoted here as 'ART initiation') are well described. ${ }^{[11-15]}$ Current specimen-based laboratory data systems are unable to provide this information because of absence of national patient identifiers to track individual patients as they progress through HCT to ART. The collection of programme data for routine $\mathrm{CD} 4$ testing on a data collection instrument using a two-tick system is proposed. This system demonstrated a significant uptake in the provision of data. The poor immune status of patients receiving their first-ever CD4 count identified by the study was a cause for concern, highlighting the challenges related to late presentation and misinterpretation of bundled CD4 data (no distinction between pre-ART v. ART). The potential of the data collection tool to assess the immunological status of patients presenting for pre-ART care across SA, once implemented, could supplement existing data collection systems that are mainly focused on ART. ${ }^{[16]}$ The value of integrating programme data collection with routine CD4 testing through a national request form lies in the ease of implementation at health facilities. The outcomes of this study indicate that it would be sustainable to implement the collection of programme data for routine CD4 testing across SA to improve management of HIV patients, either on or awaiting treatment.
Acknowledgements. We thank Mr Bahule Motlonye, Business Manager, NHLS, and Ms Nobantu Mpela, CCMT Programme Manager at the Gauteng Department of Health, Johannesburg, for her support and permission to do this work. We also thank the nursing and laboratory staff at the study sites, as well as Forms Media Independent (data collection instrument printing), Dr Helena Vreede (LIS set-up), Ms Happy Mashigo (LIS Department) and the NHLS CDW. This study forms part of the work presented for NC's MPH degree.

\section{References}

1. National Department of Health. HIV and AIDS and STI Strategic Plan for South Africa: 2007-2011. Pretoria: $\mathrm{NDoH}, 2009$

2. Rehle TM, Hallett TB, Shisana O, et al. A decline in new HIV infections in South Africa: Estimating HIV incidence from three national HIV surveys in 2002, 2005 and 2008. PloS One 2010;5(6):e11094. DOI:10.1371/journal.pone.0011094

3. National Department of Health. National Strategic Plan for HIV and AIDS, STIs and TB, 2012-2016. Pretoria: NDoH, 2011:82.

4. National Health Laboratory Service. National Health Laboratory Services Annual Report 2013/2014. Johannesburg: NHLS, 2014. http://www.nhls.ac.za (accessed 15 June 2015).

5. National Department of Health. The 2012 National Antenatal Sentinel HIV and Herpes Simplex Type2 Prevalence Survey, South Africa. Pretoria: $\mathrm{NDoH}, 2012$.

6. National Department of Health. National Consolidated Guidelines for the Prevention of Motherto-child Transmission of HIV (PMTCT) and the Management of HIV in Children, Adolescents and Adults. Pretoria: NDoH, 2015

7. Lamb MR, Eduardo E, Kandula S, et al. HIV clinical and program outcomes among older patients with HIV enrolled in HIV care and initiating ART in sub-Saharan Africa. Presented at the XIX
wition International AIDS Conference, Washington, DC, USA, 27 July 2012. Oral abstract. http://pag. aids2012.org/Abstracts.aspx?AID=21268 (accessed 7 March 2016)

8. World Health Organization. WHO Case Definition of HIV for Surveillance and Revised Clinical Staging and Immunological Classification of HIV-related Disease in Adults and Children. 2007. Geneva: WHO. http://www.who.int/hiv/pub/guidelines/HIVstaging150307.pdf (accessed 7 March 2016).

9. Glencross DK, Janossy G, Coetzee LM, et al. CD8/CD38 activation yields important clinical information of effective antiretroviral therapy: Findings from the first year of the CIPRA-SA cohort. Cytometry B Clin Cytom 2008;74(Suppl 1):S131-S140. DOI:10.1002/cyto.b.20391

10. Department of Social Development. Old Age Grant. Pretoria: DSD, 2008.

11. Fox MP, Sanne IM, Conradie F, et al. Initiating patients on antiretroviral therapy at CD4 cell counts above $200 \mathrm{cells} / \mathrm{microl}$ is associated with improved treatment outcomes in South Africa. AIDS 2010;24(13):2041-2050. DOI:10.1097/QAD.0b013e32833c703e

12. Fox MP, Shearer K, Maskew M, et al. Treatment outcomes after 7 years of public-sector HIV treatment. AIDS 2012;26(14):1823-1828. DOI:10.1097/QAD.0b013e328357058a

13. Larson BA, Brennan A, McNamara L, et al. Early loss to follow up after enrolment in pre-ART care ar. Larson BA, Brennan A, McNamara L, et al. Early loss to follow up after enrolment in pre-ART care
at a large public clinic in Johannesburg, South Africa. Trop Med Int Health 2010;15(Suppl 1):43-47. DOI:10.1111/j.1365-3156.2010.02511.x

14. Larson BA, Brennan A, McNamara L, et al. Lost opportunities to complete CD4+ lymphocyte testing among patients who tested positive for HIV in South Africa. Bull World Health Organ 2010;88(9):675680. DOI:10.2471/BLT.09.068981

15. Clouse K, Pettifor AE, Maskew M, et al. Patient retention from HIV diagnosis through one year on antiretroviral therapy at a primary health care clinic in Johannesburg, South Africa. J Acquir Immune Defic Syndr 2013;62(2):e39-e46. DOI:10.1097/QAI.0b013e318273ac48

16. University of Washington Global Health Start Program. Paper Health Registers Project Case Study: South Africass 3-Tiered Antiretroviral Treatment Monitoring System. Report to the Bill and Melinda Gates Foundation. United States of America: 2014. https://docs.gatesfoundation org/Documents/ START_43_HealthRegisters_GhanaCaseStudy_FINAL_2014-05-27.pdf (accessed 7 March 2016).

Accepted 17 December 2015 